\title{
Response to Trans-arterial Radioembolization Therapy for Liver Cancer According to Voxel-Based Dosimetry: A Retrospective Study
}

\section{Min Young Yoo}

Chunbuk National University Hospital https://orcid.org/0000-0002-6360-1108

Jin Chul Paeng ( $\square$ paengjc@snu.ac.kr)

Seoul National University Hospital https://orcid.org/0000-0002-7464-9342

Hyo-Cheol Kim

Seoul National University Hospital

Min Sun Lee

Korea Atomic Energy Research Institute

Jae Sung Lee

Seoul National University College of Medicine

Dong Soo Lee

Seoul National University Hospital

Keon Wook Kang

Seoul National University Hospital

Gi Jeong Cheon

Seoul National University Hospital

Original research

Keywords: voxel S values, 90Y-microspheres, hepatocellular carcinoma, radioembolization, prognosis

Posted Date: October 30th, 2020

DOI: https://doi.org/10.21203/rs.3.rs-98377/v1

License: (1) (1) This work is licensed under a Creative Commons Attribution 4.0 International License.

Read Full License 


\section{Abstract}

\section{Background}

Usual clinical dosimetry models for trans-arterial radioembolization (TARE) is based on assumption of uniform dose distribution in each tissue compartment. We performed a simple voxel-based dosimetry using post-treatment yttrium-90 $\left({ }^{90} \mathrm{Y}\right)$ microsphere PET in TARE and investigated the prognostic value of dosimetry parameters from the voxel-based dosimetry.

\section{Method}

Twenty-eight patients with hepatocellular carcinoma who underwent TARE using ${ }^{90} \mathrm{Y}$-microsphere were retrospectively included. Mean absorbed dose of each lesion (TDv) was analyzed using voxel-based dose maps derived from posttreatment ${ }^{90} \mathrm{Y}$-microsphere PET and voxel-wise S-value kernels. Heterogeneity of intra-tumoral absorbed dose was investigated using standard deviation and coefficient of variation of voxel doses in a tumor. The response of each lesion was classified as local control success (LCS) and local control failure (LCF) based on follow-up MRI or CT. Prognostic values of dosimetry parameters and clinicopathologic factors were evaluated using survival analysis for progression-free survival.

\section{Results}

Fourteen lesions from 10 patients were analyzed. The LCS and LCF groups showed significant differences in TDv and intra-tumoral absorbed dose heterogeneity, but not in the tumor size. Univariate survival analysis identified high serum aspartate transaminase level (> $40 \mathrm{IU} / \mathrm{L})$, large tumor size (> $66 \mathrm{~mm}$ ), and low TDv (<81 Gy) as significant prognostic factors. However, on multivariate analysis, only low TDv was identified as an independent predictive factor $(P=0.022 ; \mathrm{HR}, 21.018 ; 95 \% \mathrm{Cl}: 1.549-$ $258.204)$, with a cutoff value of $80-120 \mathrm{~Gy}$. Spearman's correlation analysis also showed a significant correlation between TDv and PFS $(P=0.009, r=0.669)$.

\section{Conclusions}

In TARE using ${ }^{90} \mathrm{Y}$-microspheres, voxel-wise absorbed dose can be easily estimated on post-treatment ${ }^{90} \mathrm{Y}$ PET using a simple method. The TDv calculated by the voxel-based dosimetry is an effective prognostic factor for PFS with a cutoff value of 80-120 Gy.

\section{Background}

Hepatocellular carcinoma (HCC) is a highly lethal malignancy prevalent worldwide [1]. Among the treatment options for $\mathrm{HCC}$, complete resection or liver transplantation are the only curative treatments. Trans-arterial radioembolization (TARE) is currently used as a bridging treatment or a local control method for patients with intermediate-risk or advanced HCC who are not eligible for curative treatments $[2,3]$. TARE has shown better survival outcomes than other treatment options, such as trans-arterial 
chemoembolization (TACE) and systemic chemotherapy including sorafenib [4]. However, local control failure (LCF) is observed in approximately $20-50 \%$ of cases [4, 5], probably because of insufficient radiation dose to tumors.

Usual dosimetry models for TARE estimate the mean absorbed radiation doses of tumors, normal liver tissues supplied by tumor-feeding artery (in-target normal liver), and normal liver tissues supplied by nontumor-feeding arteries (out-target normal liver) assuming a uniform dose distribution in each tissue compartment $[6,7]$. The radiation dose delivered by TARE is determined using a partition model of dosimetry based on pretreatment technetium-99m ( ${ }^{99 \mathrm{~m}} \mathrm{Tc}$ ) macroaggregated albumin (MAA) scan or simply based on tumor volume. However, there is a considerable intra-tumoral heterogeneity in the actual dose distributions depending on vessel density, thrombosis, and tumor necrosis. Hence, intra-tumoral dose heterogeneity is considered an underlying cause of LCF [8].

Voxel-based dosimetry was developed as a method to overcome the issue of intra-tumoral dose heterogeneity. Dieudonné et al. reported the feasibility of voxel-based three-dimensional (3D) dose mapping based on ${ }^{99 m}$ Tc-MAA single-photon emission computed tomography (SPECT)/computed tomography (CT) performed for pretreatment planning of yttrium-90 $\left({ }^{90} \mathrm{Y}\right)$ microsphere TARE [9]. Kafrouni et al. demonstrated the correlation between treatment response and mean absorbed radiation dose derived from voxel-based dosimetry based on post-treatment ${ }^{90} \mathrm{Y}$-microsphere positron emission tomography (PET) [10].

In this study, voxel-based dosimetry was performed using posttreatment ${ }^{90} \mathrm{Y}$-microsphere PET in those who underwent TARE for HCC. This study aimed to investigate the prognostic value of radiation dose parameters obtained from voxel-based dosimetry and other clinical factors in predicting the treatment outcomes of TARE.

\section{Methods}

\section{Patients}

This study retrospectively included patients who underwent TARE from July 2012 to September 2014 based on the following inclusion criteria: (1) confirmed HCC diagnosis, (2) presence of post-TARE ${ }^{90} \mathrm{Y}$ microsphere PET data, and (3) presence of follow-up records and imaging studies for evaluating treatment response. The clinicopathologic information of each patient was obtained by reviewing their electronic medical records. The Institutional Review Board of Seoul National University Hospital approved the study design and waived the requirement for informed consent (H-2009-114-1158).

\section{TARE and PET Image Acquisition}

TARE was performed as previously described [11]. During planning angiography, $185 \mathrm{MBq}$ of ${ }^{99 \mathrm{~m}} \mathrm{Tc}-\mathrm{MAA}$ was injected into the tumor-feeding artery. Subsequently, planar scan and SPECT/CT were performed to 
calculate the liver-to-lung shunt fraction (LSF) and dose absorbed by normal organs and tumors. TARE was performed when the LSF on the planar scan was $<20 \%$. The dose of injected radioactivity was determined using partition model-based dosimetry with ${ }^{99 m}$ Tc-MAA SPECT/CT [6], so that estimated tumor dose is >120 Gy. Radioactivity was adjusted, if necessary, according to the patient's condition and the operator's decision. TARE was performed by a single experienced interventional radiologist (H.C.K.) using ${ }^{90}$ Y-labeled resin microspheres (SIR-Spheres ${ }^{\circledR}$; Sirtex Medical Ltd., Lane Cove, Australia).

${ }^{90} \mathrm{Y}$-microsphere PET/CT was performed using a large field-of-view PET/CT scanner (Biograph mCT64, Siemens Healthineers, Germany; axial field-of-view $216 \mathrm{~mm}$ ) immediately after TARE. CT images were acquired first (slice thickness, $5 \mathrm{~mm}$; pitch, 1.2, $120 \mathrm{kVp}$; and $35 \mathrm{mAs}$ ) for attenuation correction and lesion localization. PET images were acquired using a 3D mode for one bed position to cover the lower chest and upper abdomen for $10 \mathrm{~min}$. The images were reconstructed on $200 \cdot 200$ matrices using an iterative method ( 2 iterations, 21 subsets) with an algorithms for point-spread function recovery and timeof-flight estimation.

\section{Voxel-based Dosimetry}

The voxel S-value (VSV) kernel [Gy/MBq•sec] convolution approach was used, where VSV is a voxel-level Medical Internal Radiation Dose (MIRD) schema, defined as the mean absorbed dose to a target voxel per radioactive decay in a source voxel [12]. The voxel dose $(D)$ was calculated by convolving the cumulated activity $(\widetilde{A})$ with VSV using the equation below.

$$
D=\tilde{A} * V S V=\sum_{i=0}^{n} \tilde{A}_{\text {voxel }_{i}} \cdot V S V\left(\text { voxel }_{j} \leftarrow \text { voxel }_{i}\right)
$$

Voxel-based dosimetry was conducted using VSV the kernel reported in a public database [13]. The $6 \cdot 6$. 6 VSV kernel of ${ }^{90} \mathrm{Y}$ was used with the voxel size of $3 \cdot 3 \cdot 3 \mathrm{~mm}^{3}$. The PET images were re-sized such that their voxel size is same as that of the VSV kernel to avoid false dose calculation. Accumulated activity maps were generated from the re-sized PET images by calculating the time-integrated activity from the injected time point to infinity. The 3D cumulated activity maps $[\mathrm{MBq} \bullet \mathrm{sec}]$ were convoluted with the 3D VSV [Gy/MBq•sec] to yield absorbed dose maps [Gy] using MATLAB software.

These absorbed dose maps were fused with enhanced magnetic resonance (MR) or CT images obtained immediately before TARE, using a vendor-supplied software package (Syngo.via, Siemens Healthineers, Germany). The tumor regions-of-interest were drawn manually based on enhanced MR or CT images on every image slice, and the tumor volume-of-interest (VOI) was obtained by stacking all tumor regions. From the VOI on voxel-based dose map, the mean absorbed tumor dose (TDv), standard deviation (SD), and coefficient of variation (CV) of absorbed doses were measured for each tumor. Tumors smaller than 
$1 \mathrm{~cm}$ were excluded because of possible partial-volume effects. A representative image of a tumor VOI is shown in Fig. 1.

\section{Response Evaluation and Statistical Analysis}

Tumor response was determined during follow-up imaging studies, including enhanced MR imaging (MRI) and/or CT. The local response of each lesion was determined according to the modified Response Evaluation Criteria in Solid Tumors (mRECIST) criteria based on the European Association for the Study of the Liver (EASL) guidelines [14-16]. Complete remission was defined as local control success (LCS), with other responses defined as local control failure (LCF). Progression was analyzed per-lesion and progression-free survival (PFS) was defined as the time from the date of TARE to the date of the imaging study that showed local control failure or progression.

Values are expressed as means \pm SDs. The median values of the longest diameter and TDv of each tumor were used as the cutoff values for survival analysis. For laboratory data, the upper normal limit of each parameter was used as the cutoff value. Univariate and multivariate survival analyses were performed using the Kaplan-Meier method and Cox regression analysis, respectively. The hazard ratio (HRs) and $95 \%$ confidence intervals (Cls) were also calculated. Group comparisons of values were performed using Mann-Whitney $\mathrm{U}$ tests. SD and CV were used as heterogeneity indices in the VOI. Correlations between factors were assessed using Spearman's correlation analysis. $P$-values less than 0.05 were considered statistically significant.

\section{Results}

\section{Patients and Treatment Responses}

During the study period, 28 patients underwent TARE in our institution. Among them, 13 had HCC, and 10 (all men; age, $59 \pm 10$ years; range, $48-84$ years) had available data and were finally included in the analysis. The patient characteristics are summarized in Table 1. No severe procedure-related complications after TARE were reported in any of the patients. The analysis included a total of 14 measurable lesions from the 10 patients; four patients had two lesions each. The average tumor size was $71.0 \pm 40.7 \mathrm{~mm}$. 
Table 1

Patient characteristics

\begin{tabular}{|c|c|c|}
\hline Variables & & Values (range) \\
\hline Age, years & & $56(48-84)^{\star}$ \\
\hline \multirow[t]{2}{*}{ Sex } & Male & 10 \\
\hline & Female & 0 \\
\hline Baseline AFP (ng/mL) & & $359.5(1.6-123000)^{*}$ \\
\hline Baseline PIVKA-II (mAU/ml) & & $1621.0(21.0-75000)^{\star}$ \\
\hline \multirow[t]{2}{*}{ Child-Pugh class } & $A$ & 9 \\
\hline & $\mathrm{B}$ & 1 \\
\hline \multirow[t]{3}{*}{ Viral status } & HBV & 7 \\
\hline & $\mathrm{HCV}$ & 1 \\
\hline & NBNC & 2 \\
\hline ECOG status & 0 & 10 \\
\hline \multirow[t]{2}{*}{ Portal vein thrombosis } & Presence & 6 \\
\hline & Absence & 4 \\
\hline
\end{tabular}

\section{Treatment Response and Voxel-Based Dosimetry}

The median follow-up duration was 2.8 months (range, 1.0-29.7 months). Among 14 lesions, LCS was achieved in five (36\%) for a median duration of 25.6 months (range, 21.9-29.7 months). LCF was observed in the other nine lesions (64.3\%) after a median follow-up of 1.5 months (range, 1.0-4.4 months). The tumor size was larger in the LCF than that in the LCS group but the difference was not statistically significant $(83.7 \pm 45.1 \mathrm{~mm} v$ s. $48.2 \pm 26.7 \mathrm{~mm}$, Fig. $2 \mathrm{~A})(P=0.266)$.

Dose maps were successfully generated using the VSV kernel convolution (Fig. 1). The average lesion TDv was $93.2 \pm 45.9 \mathrm{~Gy}$ (range, 8.8-177.2 Gy). The TDv was significantly higher in the LCS group (132.3 $\pm 13.1 \mathrm{~Gy}$; range, $117.2-150.9 \mathrm{~Gy})$ than that in the LCF group (71.5 $\pm 43.3 \mathrm{~Gy}$; range, 8.8-177.2 Gy) (Fig. 2B, $P=0.021$ ). There were no significant difference in the heterogeneity indices of absorbed doses between the two groups (SD: $P=0.266$; CV: $P=1.000$ ) (Supplemental Fig. 1). 


\section{Voxel-based Dosimetry and Survival}

Univariate survival analyses conducted by including tumor factors and clinicopathologic factors showed that high serum aspartate transaminase (AST) level ( $>40 \mathrm{IU} / \mathrm{L})$, large tumor size $(>66 \mathrm{~mm})$, and low TDv $(<81 \mathrm{~Gy})$ were significant prognostic factors for poor PFS $(P=0.019,0.042$, and 0.026 , respectively; Table 2). The cutoff values for tumor size and TDv were $66 \mathrm{~mm}$ and $81 \mathrm{~Gy}$, respectively. Kaplan-Meier survival curves of these factors are shown in Fig. 3. Multivariate analysis conducted by including these factors identified only TDv as an independent predictive factor for PFS $(P=0.022 ; \mathrm{HR}, 21.018 ; 95 \% \mathrm{Cl}$ : 1.549-285.204). TDv and PFS showed significant correlations $(r=0.669, P=0.009)$ and five of six patients (83\%) with TDv > 81 Gy showed PFS > 20 months (Fig. 4). 
Table 2

Results of survival analysis for PFS

\begin{tabular}{ll} 
Variables & Univariate analysis \\
\cline { 2 - 2 } & $\begin{array}{l}\text { Hazard ratio (95\% } \\
\mathrm{Cl})\end{array}$
\end{tabular}

Age (years)

$<65$

$\geq 65$

Portal vein thrombosis

Absence

Presence

LC type

$\mathrm{HBV} / \mathrm{HCV}$

NBNC

AST (IU/L)

$<40$

$\geq 40$

ALT (IU/L)

$<40$

$\geq 40$

Total bilirubin $(\mathrm{mg} / \mathrm{dL})$

$<1.2$

$\geq 1.2$

Size $(\mathrm{mm})$

$<66$

$\mathrm{Cl}$; confidence interval; PFS, progression-free survival; LC, liver cirrhosis; HBV, hepatitis B virus; $\mathrm{HCV}$, hepatitis C virus; NBNC, patients with neither hepatitis B nor hepatitis C; AST, aspartate transaminase; ALT, alanine transaminase
Multivariate analysis

$P \quad$ Hazard ratio

value

$(95 \% \mathrm{Cl})$

$P$

value

0.974

1.000

$1.026(0.209-$

5.031)

0.301

1.000

2.008 (0.495-

$8.155)$

0.975

1.000

1.026 (0.209-

5.031)

0.019

0.289

1.000

5.367 (1.078-

26.727)

0.267

1.000

7.678)

0.267

1.000

2.034 (0.539-

7.678)

0.042

0.506

1.00
2.034 (0.539- 


\begin{tabular}{|c|c|c|c|c|}
\hline \multirow[t]{2}{*}{ Variables } & \multicolumn{2}{|l|}{ Univariate analysis } & \multicolumn{2}{|c|}{ Multivariate analysis } \\
\hline & $\begin{array}{l}\text { Hazard ratio }(95 \% \\
\mathrm{Cl})\end{array}$ & $\begin{array}{l}P \\
\text { value }\end{array}$ & $\begin{array}{l}\text { Hazard ratio } \\
(95 \% \mathrm{Cl})\end{array}$ & $\begin{array}{l}P \\
\text { value }\end{array}$ \\
\hline$\geq 66$ & $\begin{array}{l}3.741(0.915- \\
15.290)\end{array}$ & & & \\
\hline $\begin{array}{l}\text { Absorbed dose based on dose } \\
\text { map (Gy) }\end{array}$ & & 0.026 & & 0.022 \\
\hline$<81$ & $\begin{array}{l}11.187(1.341- \\
93.342)\end{array}$ & & $\begin{array}{l}21.018(1.549- \\
285.204)\end{array}$ & \\
\hline$\geq 81$ & 1.00 & & 1.00 & \\
\hline \multicolumn{5}{|c|}{$\begin{array}{l}\text { Cl; confidence interval; PFS, progression-free survival; LC, liver cirrhosis; HBV, hepatitis B virus; HCV, } \\
\text { hepatitis C virus; NBNC, patients with neither hepatitis B nor hepatitis C; AST, aspartate transaminase; } \\
\text { ALT, alanine transaminase }\end{array}$} \\
\hline
\end{tabular}

\section{Discussion}

TARE is an effective treatment option for intermediate- or advanced-stage HCC or in cases of portal vein thrombosis. In addition to the low complication rate, the reported outcomes of TARE are comparable to or even better than those of other treatment options such as TACE or systemic chemotherapy [17-20]. In TARE, radiopharmaceuticals are directly injected into the tumor-feeding arteries and do not show redistribution after initial embolization. Thus, calculation of its treatment dose is easier than that of other systemically administered radioactive drugs. Pretreatment planning angiography is currently performed using ${ }^{99 m}$ Tc-MAA to simulate radiation doses to the tumor, normal liver tissues, and lungs.

In addition to pretreatment scans, many institutions perform a post-treatment scan to assess the results of TARE and identify possible complications occurring because of exposure of unexpected organs to radioactivity. Because ${ }^{90} \mathrm{Y}$ is a pure beta-emitter, the post-treatment scans are performed using highly sensitive scanners to acquire bremsstrahlung gamma images or PET images. The radiation dosimetry methods using post-treatment images are based on the MIRD schema. Several studies have applied the MIRD schema to bremsstrahlung imaging [21,22], and Gulec et al. reported the use of this schema for the dosimetry of ${ }^{90} \mathrm{Y}$-microspheres confined to the liver [23]. The usual MIRD schema assumes a uniform distribution of radioactivity in a certain organ or tissues and its use is very simple.

However, in the real world, therapeutic radiopharmaceuticals show heterogeneous distributions in-target tissues or organs because of vasculature heterogeneity, anatomical variation, and tissue necrosis. This study adopted voxel-based dosimetry, which considered intra-tumoral heterogeneity of ${ }^{90} \mathrm{Y}$-microsphere distribution [24]. In this voxel-based dosimetry study, the VSV was calculated for each voxel to create an absorbed dose map. This is an easier approach than that involving patient-specific Monte Carlo 
simulations and showed accurate dosimetry results in uniform-density organs such as the liver [24]. We applied a VSV from a ${ }^{90} \mathrm{Y}$ dataset published previously [13].

Based on the voxel-based dose map, the TDv of each lesion was successfully calculated. The considerable variation in TDv, from $40 \mathrm{~Gy}$ to $177 \mathrm{~Gy}$, may be the main reason behind the difference in treatment outcomes. In survival analysis, small tumor size, low AST level, and low TDv (median, $\geq 81 \mathrm{~Gy}$ ) were significant prognostic factors for successful treatment. The multivariate analysis confirmed TDv as the only independent prognostic factor. Previous studies reported clinical factors related to hepatic function (total bilirubin, albumin) and tumor aggressiveness (alpha-fetoprotein level, portal vein thrombosis, and tumor size) as significant prognostic factors in patients treated with TARE $[25,26]$. In our study, these factors were not significant, probably because of the small sample size. Further investigation in a larger cohort is needed to determine the role of these factors.

When applying a partition model of dosimetry to pretreatment simulation scans, 120 Gy is deemed a target dose for effective treatment. In our study, some lesions were outside this target range. Kao et al. reported that HCC tumors receiving > 105 Gy showed a higher response rate when the tumor dose was calculated using a partition model-based dosimetry model on ${ }^{99 m}$ Tc-MAA SPECT/CT [27]. Kokabi et al. estimated the tumor dose using ${ }^{90} \mathrm{Y}$ bremsstrahlung SPECT/CT and reporting a significant correlation between a tumor dose of $>105 \mathrm{~Gy}$ and prolonged survival [28]. We observed an obvious difference in PFS between groups showing high and low absorbed doses. In Fig. 4, the cutoff of the TDv was set at 80$120 \mathrm{~Gy}$. We also observed significant correlations between the absorbed dose and PFS in correlation analysis; this finding is consistent with that of a previous study [29].

Because this study performed voxel-based dosimetry, it was possible to calculate the voxel-wise intratumoral heterogeneity. High intra-tumoral heterogeneity was assumed to be related to LCF, despite the high average tumor dose. The SD and CV of dose distributions in a tumor were measured as simple indices for heterogeneity [30]. Although there was no significant difference in these values between the LCS and LCF groups in our study, further studies including more cases are warranted to investigate the effects of intra-tumoral dose distributions.

This study has several limitations. First, a small number of patients with inoperable HCC who underwent a single TARE session with ${ }^{90} \mathrm{Y}$-resin microspheres were included in this study. Further studies with large cohorts are required to investigate the role of voxel-based dosimetry in clinical practice. Second, we used only simple indices for intra-tumoral heterogeneity. However, the results of this study may be used as preliminary data for determining the role of intra-tumoral heterogeneity of absorbed doses in treatment response.

\section{Conclusions}

In TARE using ${ }^{90} \mathrm{Y}$-microsphere, the voxel-wise absorbed dose can be easily estimated using posttreatment ${ }^{90} \mathrm{Y}$ PET with a simple voxel-based dosimetry method conducted using VSV kernels. The TDV 
calculated by voxel-based dosimetry was a significant prognostic factor for the outcome of TARE in patients with HCC, with a cutoff value of 80-120 Gy. Further studies are required to determine the roles of tumor dose and intra-tumoral heterogeneity in treatment response.

\section{Abbreviations}

AST: aspartate transaminase

Cl: Confidence interval

CT: Computed tomography

CV: Coefficient of variation

EASL: European Association for the Study of the Liver

HCC: Hepatocellular carcinoma

HR: Hazard ratio

LCF: Local control failure

LCS: Local control success

MAA: Macroaggregated albumin

MIRD: Medical Internal Radiation Dose

mRECIST: modified Response Evaluation Criteria in Solid Tumors

PET: Positron emission tomography

PFS: Progression-free survival

SD: Standard deviation

SPECT: Single-photon emission computed tomography

TACE: Trans-arterial chemoembolization

TARE: Trans-arterial radioembolization

TDv: Mean absorbed tumor dose

VOI: Volume-of-interest

VSV: Voxel S-value 


\section{Declarations}

\section{Ethics approval and consent to participate}

This study was reviewed and approved by the Institutional Review Board of Seoul National University Hospital. Requirement for written informed consent was waived for the retrospective cohort.

\section{Consent for publication}

Not applicable.

\section{Availability of data and material}

The datasets used and/or analyzed during the current study are available from the corresponding author upon reasonable request.

\section{Competing interests}

The authors declare that they have no competing interests

\section{Funding}

This work was supported by grants from the National Research Foundation of Korea (NRF) funded by the Korean Ministry of Science and ICT (grant no. NRF- 2020M2D9A109398911).

\section{Authors' contributions}

JCP, JSL, MSL, and MYY designed the study and drafted the manuscript. JSL, MSL, and MYY performed the analysis and interpreted the results. JCP and JSL revised the manuscript. HCK, KWK, DSL, and GJC collected clinical data. All authors have read and approved the final manuscript.

\section{Acknowledgments}

Not applicable

\section{References}

1. El-Serag HB, Rudolph KL. Hepatocellular carcinoma: epidemiology and molecular carcinogenesis. Gastroenterology. 2007;132:2557-76. 
2. Jelic S, Sotiropoulos GC, Group EGW. Hepatocellular carcinoma: ESMO Clinical Practice Guidelines for diagnosis, treatment and follow-up. Ann Oncol. 2010;21:v59-64.

3. Thomas MB, Jaffe D, Choti MM, Belghiti J, Curley S, Fong Y, et al. Hepatocellular carcinoma: consensus recommendations of the National Cancer Institute Clinical Trials Planning Meeting. J Clin Oncol. 2010;28:3994-4005.

4. Moreno-Luna LE, Yang JD, Sanchez W, Paz-Fumagalli R, Harnois DM, Mettler TA, et al. Efficacy and safety of transarterial radioembolization versus chemoembolization in patients with hepatocellular carcinoma. Cardiovasc Intervent Radiol. 2013;36:714-23.

5. Lewandowski R, Kulik L, Riaz A, Senthilnathan S, Mulcahy M, Ryu R, et al. A comparative analysis of transarterial downstaging for hepatocellular carcinoma: chemoembolization versus radioembolization. Am J Transplant. 2009;9:1920-8.

6. Dezarn WA, Cessna JT, DeWerd LA, Feng W, Gates VL, Halama J, et al. Recommendations of the American Association of Physicists in Medicine on dosimetry, imaging, and quality assurance procedures for $90 \mathrm{Y}$ microsphere brachytherapy in the treatment of hepatic malignancies. Med Phys. 2011;38:4824-45.

7. Loevinger R, Budinger T, Watson E. MIRD primer for absorbed dose calculations. Revised ed. New York City: The Society of Nuclear Medicine, Inc.; 1991.

8. Sangro B, Iñarrairaegui M, Bilbao JI. Radioembolization for hepatocellular carcinoma. J Hepatol. 2012;56:464-73.

9. Dieudonné A, Garin E, Laffont S, Rolland Y, Lebtahi R, Leguludec D, et al. Clinical feasibility of fast 3dimensional dosimetry of the liver for treatment planning of hepatocellular carcinoma with $90 \mathrm{Y}$ microspheres. J Nucl Med. 2011;52:1930-7

10. Kafrouni M, Allimant C, Fourcade M, Vauclin S, Delicque J, llonca AD, et al. Retrospective voxel-based dosimetry for assessing the ability of the body-surface-area model to predict delivered dose and radioembolization outcome. J Nucl Med. 2018;59:1289-95.

11. Song YS, Paeng JC, Kim HC, Chung JW, Cheon GJ, Chung JK, et al. PET/CT-based dosimetry in 90Ymicrosphere selective internal radiation therapy: single cohort comparison with pretreatment planning on 99mTc-MAA imaging and correlation with treatment efficacy. Medicine. 2015;94:23.

12. Bolch W, Bouchet L, Robertson J, Wessels BW, Siegel JA, Howell RW, et al. MIRD pamphlet no. 17: the dosimetry of nonuniform activity distributions-radionuclide $S$ values at the voxel level. J Nucl Med. 1999;40:11S36S.

13. Lanconelli N, Pacilio M, Meo SL, Botta F, Di Dia A, Aroche LT, et al. A free database of radionuclide voxel S values for the dosimetry of nonuniform activity distributions. Phys Med Biol. 2012;57:517.

14. Bruix J, Sherman M, Llovet JM, Beaugrand M, Lencioni R, Burroughs AK, et al. Clinical management of hepatocellular carcinoma. Conclusions of the Barcelona-2000 EASL conference. J Hepatol. 2001;35:421-30.

15. Gillmore R, Stuart S, Kirkwood A, Hameeduddin A, Woodward N, Burroughs AK, et al. EASL and mRECIST responses are independent prognostic factors for survival in hepatocellular cancer patients 
treated with transarterial embolization. J Hepatol. 2011;55:1309-16.

16. Lencioni R, Llovet JM. Modified RECIST (mRECIST) assessment for hepatocellular carcinoma. Semin Liver Dis. 2010;30:52-60.

17. Bruix J, Sherman M. Management of hepatocellular carcinoma: an update. Hepatology. 2011;53:1020-2.

18. Salem R, Lewandowski RJ, Kulik L, Wang E, Riaz A, Ryu RK, et al. Radioembolization results in longer time-to-progression and reduced toxicity compared with chemoembolization in patients with hepatocellular carcinoma. Gastroenterology. 2011;140:497-507. e2.

19. Kulik LM, Carr BI, Mulcahy MF, Lewandowski RJ, Atassi B, Ryu RK, et al. Safety and efficacy of 90Y radiotherapy for hepatocellular carcinoma with and without portal vein thrombosis. Hepatology. 2008;47:71-81.

20. Tsochatzis EA, Germani G, Burroughs AK. Transarterial chemoembolization, transarterial chemotherapy, and intra-arterial chemotherapy for hepatocellular carcinoma treatment. Semin Oncol. 2010;37:89-93.

21. Siegel JA, Zeiger LS, Order SE, Wallner PE. Quantitative bremsstrahlung single-photon emission computed tomographic imaging: use for volume, activity, and absorbed dose calculations. Int J Radiat Oncol Biol Phys. 1995;31:953-8.

22. Siegel J, Handy D, Kopher K, Zeiger L, Order S. Therapeutic beta irradiating isotopes in bony metastasis: a technique for bremsstrahlung imaging and quantitation. Antibody Immunoconj Radiopharm. 1992;5:237-48.

23. Gulec SA, Mesoloras G, Stabin M. Dosimetric techniques in 90Y-microsphere therapy of liver cancer: the MIRD equations for dose calculations. J Nucl Med. 2006;47:1209-11.

24. Dieudonné A, Hobbs RF, Bolch WE, Sgouros G, Gardin I. Fine-resolution voxel S values for constructing absorbed dose distributions at variable voxel size. J Nucl Med. 2010;51:1600-7.

25. Bauschke A, Altendorf-Hofmann A, Freesmeyer M, Winkens T, Malessa C, Schierz J, et al. Selective internal radioembolization in nonresectable hepatocellular carcinoma. Chirurg. 2016;87:956-63.

26. Jeliazkova P, Umgelter A, Braren R, Kaissis G, Mustafa M, Einwächter H. Prognostic factors in hepatocellular carcinoma patients undergoing transarterial chemoembolization and radioembolization: a retrospective study. Eur J Gastroenterol Hepatol. 2019.

27. Kao YH, Tan AEH, Burgmans MC, Irani FG, Khoo LS, Lo RHG, et al. Image-guided personalized predictive dosimetry by artery-specific SPECT/CT partition modeling for safe and effective $90 \mathrm{Y}$ radioembolization. J Nucl Med. 2012;53:559-66.

28. Kokabi N, Galt JR, Xing M, Camacho JC, Barron BJ, Schuster DM, et al. A simple method for estimating dose delivered to hepatocellular carcinoma after yttrium-90 glass-based radioembolization therapy: preliminary results of a proof of concept study. J Vasc Interv Radiol. 2014;25:277-87.

29. Garin E, Lenoir L, Edeline J, Laffont S, Mesbah H, Porée P, et al. Boosted selective internal radiation therapy with 90 Y-loaded glass microspheres (B-SIRT) for hepatocellular carcinoma patients: a new 
personalized promising concept. Eur J Nucl Med Mol Imaging. 2013;40:1057-68.

30. Watabe $T$, Tatsumi $M$, Watabe $H$, Isohashi $K$, Kato H, Yanagawa $M$, et al. Intratumoral heterogeneity of F-18 FDG uptake differentiates between gastrointestinal stromal tumors and abdominal malignant lymphomas on PET/CT. Ann Nucl Med. 2012;26:222-7.

\section{Figures}

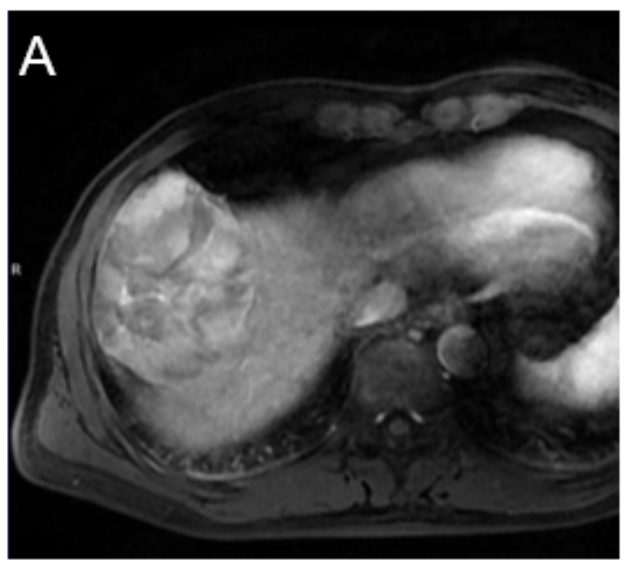

B

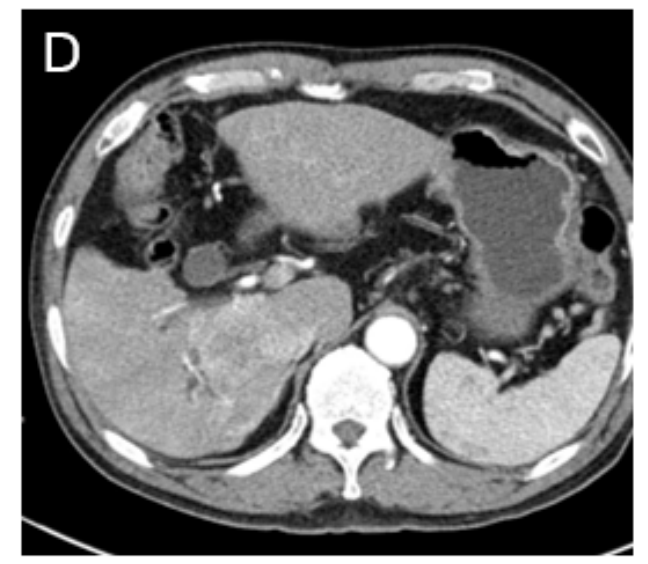

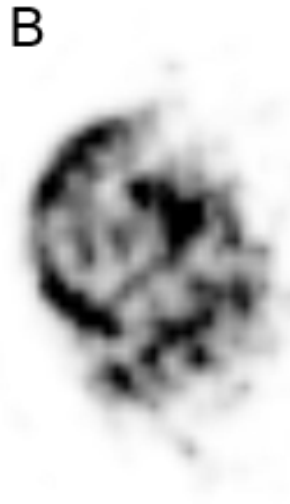

E

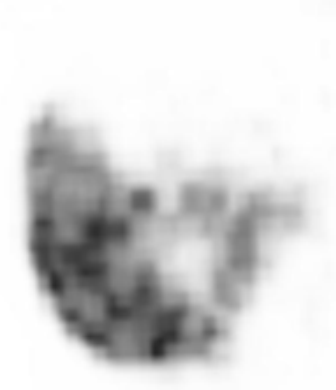

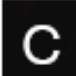

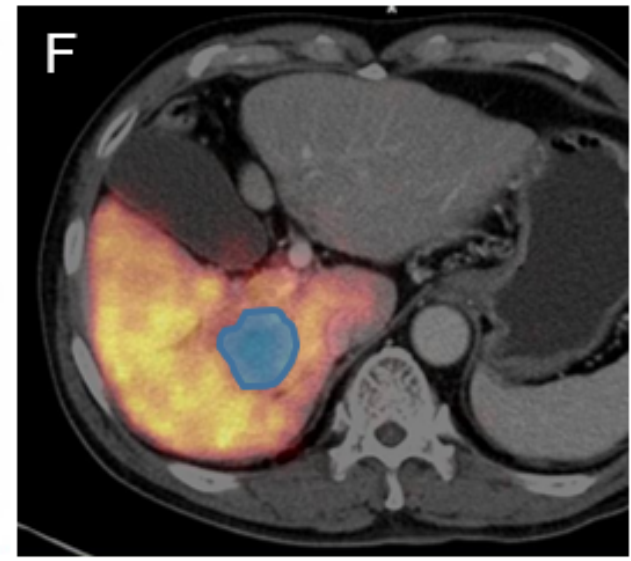

\section{Figure 1}

Representative images of patients showing local control success (LCS, top rows) and local control failure (LCF, bottom rows). In one patient, magnetic resonance imaging (MRI) showed a 92.3-mm-sized tumor (A) and the dose map obtained using 90Y positron emission tomography (PET) showed the absorption of a high dose particularly in tumor margin (B). Tumor volume-of-interest (VOI) drawn on the fusion image (C) showed a mean absorbed tumor dose (TDv) of $122.6 \mathrm{~Gy}$, and the patient demonstrated a progressionfree survival (PFS) of 29.5 months. In another patient (D), the dose map showed the absorption of a low dose in the tumor (E) and a TDv of only 54.9 Gy (F). The patient's PFS was 1.5 months. 

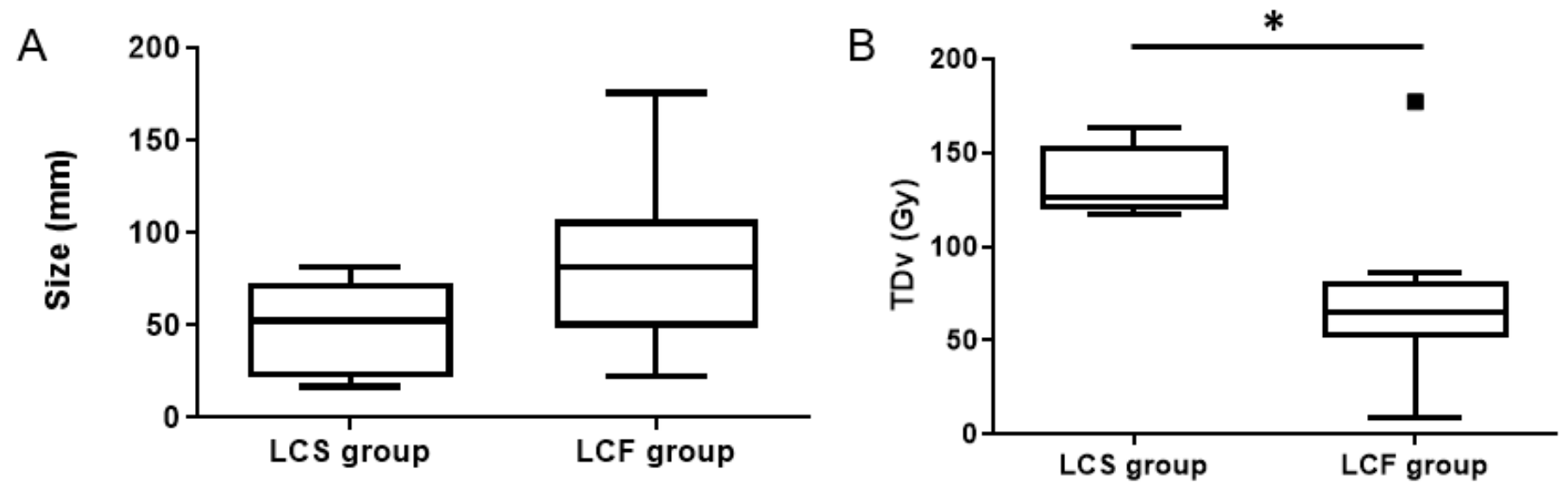

Figure 2

Box plots for tumor size and TDv according to treatment response. (A) Average tumor size was not significantly different between the local control success (LCS) and local control failure (LCF) groups $(P=$ 0.266). (B) However, the TDv was significantly higher in the LCS group than that in LCF group $(P=0.021)$.
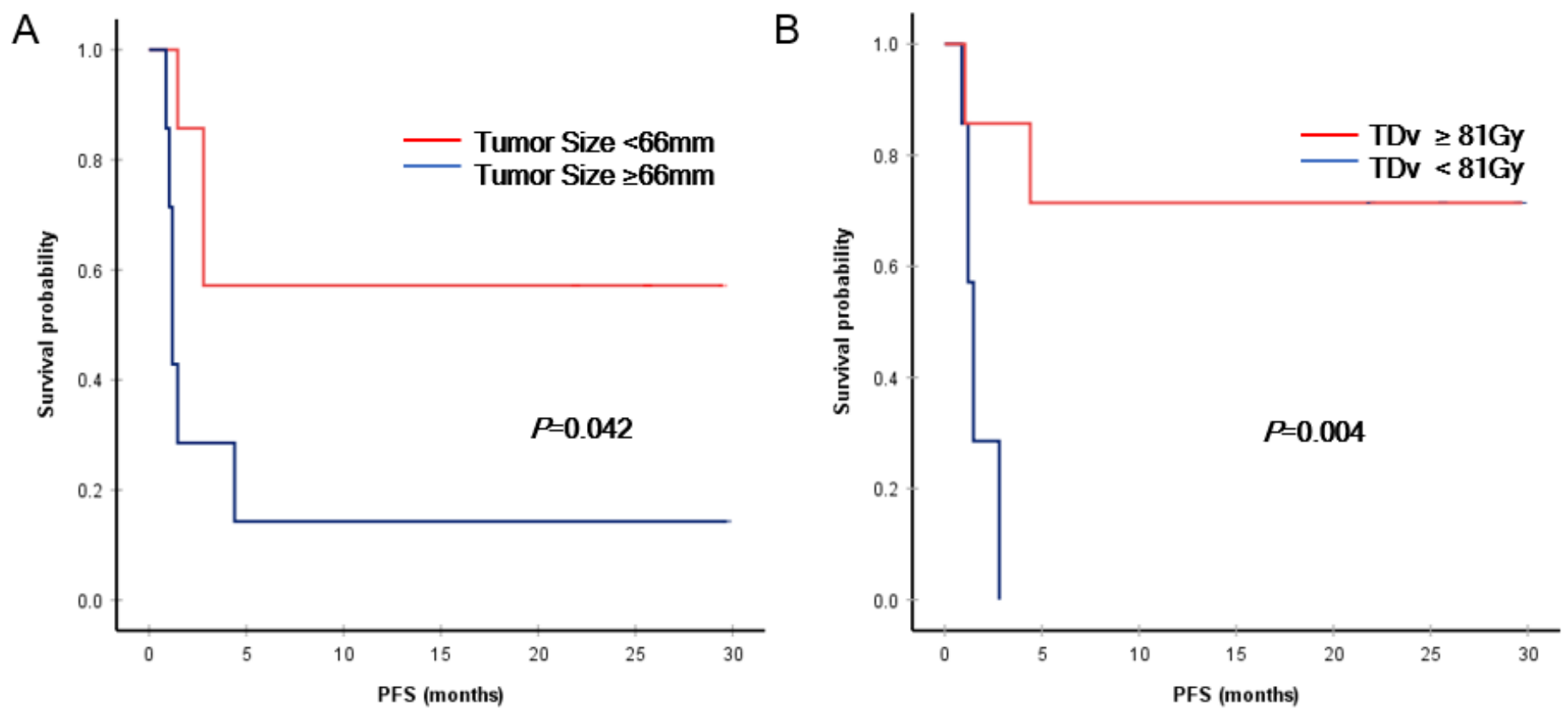

Figure 3

Kaplan-Meier survival curves showing significant differences in progression-free survival (PFS) according to tumor size (A) and TDv (B). 


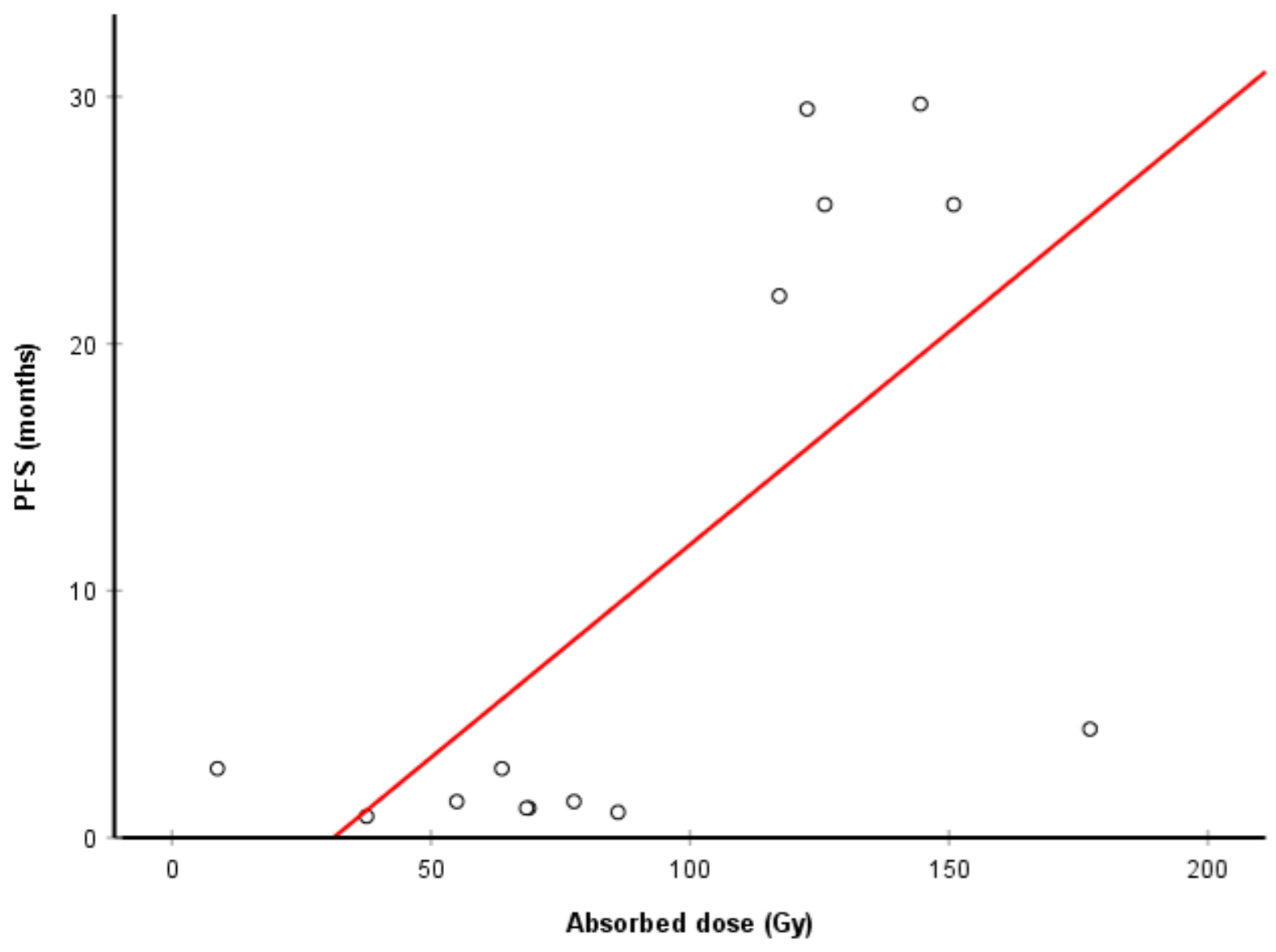

Figure 4

Correlation analysis for the relationship between mean absorbed tumor dose (TDv) and progression-free survival (PFS); significant correlation was observed $(P=0.009, r=0.669)$

\section{Supplementary Files}

This is a list of supplementary files associated with this preprint. Click to download.

- SupplementalFig.pptx 